\title{
Comparison of results of whole-body magnetic resonance imaging scans and traditional imaging modalities in diagnostic management of chronic recurrent multifocal osteomyelitis
}

Jerzy Sułko ${ }^{1}$, Artur Oberc ${ }^{2}$

\begin{abstract}
${ }^{1}$ Department of Pediatric Surgery, Jagiellonian University Medical College, Krakow, Poland

2Department of Pediatric Surgery, University Children's Hospital, Krakow, Poland
\end{abstract}

Submitted: 1 March 2018

Accepted: 15 April 2018

Arch Med Sci Civil Dis 2018; 3: e41-e46

DOI: https://doi.org/10.5114/amscd.2018.75591

Copyright @ 2018 Termedia \& Banach

\begin{abstract}
Introduction: The diagnosis of chronic recurrent multifocal osteomyelitis (CRMO) is frequently the result of excluding other diseases. The only methods that provide images of the multicentricity of radiologically active foci are whole body bone scintigraphy ( $\mathrm{SCI}$ ) and whole body MRI (WBMRI). The objective of the study was to compare the results of WBMRI and traditional imaging modalities in CRMO.

Material and methods: The study group consisted of 20 children with the mean age of 9.5 years. WBMRI imaging was performed between June and December of 2014. The mean follow-up period after completion of the study was 35 months. All the patients included in the study were additionally subjected to examination of clinically active CRMO foci by computed tomography, classic radiographic diagnostic management and scintigraphy.

Results: All the patients manifested clinically active foci. In the group of the investigated patients, the highest number of foci in concordance with the clinical picture was noted in $\mathrm{SCl}-29$, followed by CT -22 and WBMRI -21 , while plain X-ray yielded the lowest number of positive results -12 .

Conclusions: The results of the present study do not confirm the highest sensitivity of WBMRI in determining possible disease foci in CRMO as compared to other imaging modalities. Higher sensitivity in detection of radiologically active foci in CRMO was noted in bone system scintigraphy. During the 35-month follow-up, none of the WBMRI-detected radiologically active and clinically silent foci underwent clinical activation.
\end{abstract}

Key words: whole body magnetic resonance imaging, chronic recurrent multifocal osteomyelitis diagnosis, whole-body magnetic resonance imaging.

\section{Introduction}

Chronic recurrent multifocal osteomyelitis (CRMO) belongs to the group of non-bacterial osteitis (NBO). Chronic recurrent multifocal osteomyelitis is often the cause of marked diagnostic problems or else remains an undiagnosed disease entity. The diagnosis of CRMO is frequently the result of excluding other diseases when specific coexisting lesions and abnormalities are detected in imaging and laboratory studies. Unfortu-

\author{
Corresponding author: \\ Artur Oberc MD, PhD \\ Department of Pediatric \\ Surgery \\ University Children's Hospital \\ 265 Wielicka St \\ 33-663 Krakow, Poland \\ Phone: +48 602425818 \\ E-mail: artur.oberc@gmail.com
}


nately, imaging studies of CRMO-associated lesions do not provide uniform results.

Roderick et al. proposed the criteria of CRMO diagnosis termed the "Bristol diagnostic criteria for CRMO" [1]. In keeping with the above criteria, a combination of the presence of clinical activity manifested as bone pain and/or widening of the bone outline, radiological activity manifested as typical lesions in X-ray, computed tomography (CT) and magnetic resonance imaging (MRI) imaging modalities, abnormalities in laboratory tests involving C-reactive protein (CRP) and characteristic histopathological lesions in the biopsy allow for establishing the diagnosis of CRMO. It should be, however, emphasized that the histopathological findings characteristic of CRMO, namely inflammatory symptoms with the presence of lymphocytes, plasma cells, histiocytes, T and B cells, CD-68-positive macrophages and bone marrow edema, are not always present, and yet CRMO is diagnosed $[2,3]$. As stressed by Fritz et al., histopathology is significant with respect to ruling out malignancy and infectious genesis [3, 4]. On the other hand, imaging studies of single skeletal fragments with clinically active foci do not provide a picture of multifocality, which was already described by Giedon when defining CRMO [5]. As mentioned by Fritz et al., the conformity of clinical symptoms and the characteristic image of multicentricity of radiologically active foci allow for abandoning the intention of collecting a section and establishing the diagnosis of CRMO [4].

In the diagnostic management of the disease, apart from laboratory tests, the first-line imaging modality is plain X-ray involving the part of the skeletal system in which - in keeping with the patient's complaints - chronic pain is experienced or else widening of the bone outline is noted. At times, radiography does not demonstrate visible abnormalities in the bone structures. To extend the imaging diagnostic management, one may employ CT or MRI. These imaging modalities also include the region in which the patient reports pain. The only methods that provide an image of the multicentricity of radiologically active foci are whole body bone scintigraphy $(\mathrm{SCl})$ and whole body MRI (WBMRI). Scintigraphy of the bone system informs us about the activity of bone metabolism and increased isotope uptake in the painful region, but also of a possible location of foci characterized by an increased isotope uptake and situated in other regions. The $\mathrm{SCl}$ modality is at present more available and less expensive as compared to WBMRI, but it does not meet the ALARA criteria (as low as reasonably achievable) in imaging modalities in children $[4,6]$.

In recent years, the authors of reports addressing CRMO have been drawing attention to bene- fits resulting from $M R I$ involving the entire skeletal system, or the so-called whole body MRI, which may also demonstrate other disease foci. An additional asset of WBMRI is its positive evaluation in keeping with the ALARA criteria [4].

The authors decided to employ WBMRI in the patients with diagnosed CRMO they treated. The objective of the study was to assess the bone system with respect to the possible presence of other disease foci, even if they were clinically silent. All the patients included in the study were additionally subjected to examination of clinically active CRMO foci by computed tomography, classic radiographic diagnostic management and scintigraphy. In addition, the level of osteocalcin was determined in all the subjects.

The prospective study was carried out through a research grant and was issued with permission of the Bioethics Committee, Jagiellonian University Medical College (No. KBET/41/B/2014).

\section{Material and methods}

The study group consisted of 20 children at the mean age of 9.5 years (range: 2-14 years). The legal guardians of all the patients granted their informed consent to include the children in the study group. The group included 18 girls and 2 boys. All the children were diagnosed with non-bacterial osteitis (NBO), with the classic form of CRMO detected in 16 (80\%) children and synovitis acne pustulosis hyperostosis osteomyelitis (SAPHO) in 4 (20\%) patients. All the children were diagnosed in 2013 or in early 2014. WBMRI imaging was performed between June and December of 2014. The mean follow-up period after completion of the study was 35 months.

WBMRI imaging was performed using a Siemens Magnetom Avanto Tim $76 \times 18$ 1.5T in the following sequences: TSE T1, TSE T2, trufi T2 and STIR. The scans were acquired in the sagittal and frontal planes and the layer thickness was $3 \mathrm{~mm}$ and $5 \mathrm{~mm}$. The imaging study included the entire body of each patient. In keeping with the recommendations provided in the literature, no enhancement with IV contrast medium (gadolinium) administration was employed [7, 8]. None of the children required sedation. The average duration of WBMRI examination was $25 \mathrm{~min}$.

The analysis was based on descriptions of the results and on assessment of the CD-recorded images performed by the authors. The radiologist who interpreted the MRI scans did not know the location of the previously determined disease foci, but was aware of the diagnosis.

Clinically active foci were defined as pain, swelling or local warming within the bone system persisting for the past 4 weeks. On the other hand, radiologically active foci in WBMRI imaging were 
Table I. Maximal, minimal and mean number of diagnosed radiologically active foci calculated per person depending on imaging modality

\begin{tabular}{|lccc|}
\hline Imaging modality & Mean number of foci per patient & Minimal number of foci & Maximal number of foci \\
\hline X-ray & 0.6 & 0 & 2 \\
\hline CT & 1.1 & 0 & 3 \\
\hline SCl & 1.45 & 0 & 3 \\
\hline WBMRI & 1.5 & 0 & 5 \\
\hline
\end{tabular}

defined as sites with increased signal intensity in the STIR sequence with a simultaneous signal intensity decrease below the signal originating from the muscle tissue in the T1-dependent sequence. In turn, an isolated signal decrease in the T1-dependent sequence without any changes in the STIR sequence was not regarded as a radiologically active focus of CRMO. The above criteria are in accordance with the criteria proposed by Arnoldi et al. [9]. The size of the described foci was not taken into account.

The results were compared with the results of imaging studies of clinically active foci performed in the course of the previous diagnostic management: plain X-ray, computed tomography and bone system scintigraphy. The results were evaluated together with laboratory determinations of the level of osteocalcin.

The mean follow-up period of 35 months allowed for evaluating clinically silent foci with respect to their clinical presentation; the foci were visualized in WBMRI as images of radiologically active lesions.

While evaluating plain X-ray pictures and computed tomography scans, radiologically active foci were defined as regions of bone lysis, sclerotization and regions of periosteal reactions. Similar abnormalities have also been described in the course of CRMO by other authors [1]. It should be emphasized that solely clinically active foci were evaluated, since only such foci were visualized employing the above imaging modalities.

In scintiscans, determinations included the uptake of Tc-99m-labeled hydroxymethylene diphosphonate (MDP). The MDP activity varied according to age and bodyweight of the patients. The average activity on investigation was $408 \mathrm{MBq}$ (megabecquerels). None of the patients required sedation.

\section{Results}

All the patients manifested clinically active foci, with the mean number of foci per person being 3.15 ( $\min .1$; $\max .9$ ).

Depending on the employed imaging modality, variable numbers of radiologically active foci were determined. The data are presented in Table I.
The patients were analyzed with respect to the prevalence and location of clinically active foci their location is presented in Table II.

Employing the aforementioned imaging modalities, the authors managed to determine the following number of radiologically active foci concurrent with clinically active foci: X-ray -12 , $\mathrm{CT}-22, \mathrm{SCl}-29, \mathrm{WBMRI}-21$. It should be emphasized that in the case of WBMRI, the authors acquired images of nine foci that were asymptomatic both at the time of the examination and in the subsequent 30-month follow-up. According to the results reported by other authors, $18-30 \%$ of radiologically active foci detected by WBMRI were also not confirmed by clinical symptoms [1, 7, 9]. The quoted reports did not provide any data on subsequent follow-up of clinically silent and radiologically active foci in WBMRI; nevertheless, the present observations did not confirm their activation within the 35-month follow-up.

In the group of the investigated patients, the highest number of foci in concordance with the clinical picture was noted in $\mathrm{SCl}$, followed by $\mathrm{CT}$ and WBMRI, while plain X-ray yielded the lowest number of positive results. The low sensitivity of plain X-ray is also mentioned by other authors,

Table II. Prevalence and location of clinically active foci in the investigated patients

\begin{tabular}{|lc|}
\hline $\begin{array}{l}\text { Location of clinically } \\
\text { active foci }\end{array}$ & $\begin{array}{c}\text { Number of clinically } \\
\text { active foci }\end{array}$ \\
\hline Clavicle & 9 \\
\hline Knee & 8 \\
\hline Spine & 7 \\
\hline Hip & 6 \\
\hline Ankle & 4 \\
\hline Jaw & 4 \\
\hline Shoulder & 3 \\
\hline Wrist & 3 \\
\hline Foot & 3 \\
\hline Ribs & 2 \\
\hline Scapula & 1 \\
\hline
\end{tabular}


who contrast the imaging modality with the high sensitivity of WBMRI or MRI involving the localized part of the body. Nevertheless, the literature on the subject lacks studies comparing particular imaging modalities.

Table III presents the obtained data along with the division into the number of patients:

- column 2 - patients in whom radiologically inactive but clinically active foci were detected,

- column 3 - studies demonstrating single-focus radiologically active abnormalities,

- column 4 - studies demonstrating radiologically active abnormalities involving two or more foci.

At the same time, it should be emphasized that single-focus CRMO was noted in 6 patients, while two or more foci were seen in the remaining 14 children. No relationship was noted suggesting that any of the analyzed imaging modalities was superior to others in diagnostic management of single or multifocal CRMO. Of significance, however, was the fact that in multifocal disease, WBMRI demonstrated the highest number of radiologically active foci devoid of clinical activity, frequently failing to show radiological activity in clinically involved regions. When comparing two imaging modalities that visualize the entire bone system, namely $\mathrm{SCl}$ and WBMRI, the authors selected 14 patients with multifocal CRMO in order to compare the sensitivity and accuracy of imaging modalities. The results are presented in Table IV.

While analyzing the results of osteocalcin determinations in the investigated patients, the authors noted the mean osteocalcin level of $37 \mathrm{ng} /$ $\mathrm{ml}(5-82)$, with the normal value used by the laboratory being up to $13.4 \mathrm{ng} / \mathrm{ml}$.

Table III. Comparison of the number of clinically active foci that did not manifest radiological activity depending on the imaging modality

\begin{tabular}{|lccc|}
\hline Imaging modality & $\begin{array}{c}\text { Clinically active and } \\
\text { radiologically inactive foci }\end{array}$ & $\begin{array}{c}\text { Single radiologically active } \\
\text { focus }\end{array}$ & $\begin{array}{c}\text { Two or more radiologically } \\
\text { active foci }\end{array}$ \\
\hline X-ray & 10 & 8 & 2 \\
\hline CT & 5 & 9 & 6 \\
\hline SCI & 3 & 8 & 9 \\
\hline WBMRI & 7 & 8 & 5 \\
\hline
\end{tabular}

Table IV. Group of patients with multicenter CRMO compared with respect to the number of clinically and radiologically active foci in $\mathrm{SCl}$ and WBMRI

\begin{tabular}{|c|c|c|c|c|c|c|}
\hline $\begin{array}{l}\text { No. of } \\
\text { patient }\end{array}$ & $\begin{array}{c}\text { Number of } \\
\text { clinically active } \\
\text { foci }\end{array}$ & $\begin{array}{l}\text { Number of } \\
\text { radiologically } \\
\text { active foci } \\
\text { in } \mathrm{SCl}\end{array}$ & $\begin{array}{c}\mathrm{SCl} \text { sensitivity } \\
(\%)\end{array}$ & $\begin{array}{l}\text { Number of } \\
\text { radiologically } \\
\text { active foci } \\
\text { in WBMRI }\end{array}$ & $\begin{array}{c}\text { WBMRI } \\
\text { sensitivity (\%) }\end{array}$ & $\begin{array}{c}\text { Falsely } \\
\text { positive foci in } \\
\text { WBMRI }\end{array}$ \\
\hline 1 & 3 & 3 & 100 & 2 & 66 & + \\
\hline 2 & 4 & 3 & 75 & 0 & 0 & - \\
\hline 3 & 3 & 3 & 100 & 1 & 33 & - \\
\hline 4 & 2 & 2 & 100 & 1 & 50 & + \\
\hline 5 & 2 & 2 & 100 & 2 & 100 & - \\
\hline 6 & 3 & 1 & 33 & 1 & 33 & - \\
\hline 7 & 4 & 3 & 75 & 4 & 100 & + \\
\hline 8 & 9 & 2 & 22 & 2 & 22 & + \\
\hline 9 & 2 & 0 & 0 & 0 & 0 & - \\
\hline 10 & 5 & 1 & 20 & 1 & 20 & - \\
\hline 11 & 4 & 2 & 50 & 3 & 75 & - \\
\hline 12 & 7 & 1 & 14 & 0 & 0 & - \\
\hline 13 & 6 & 3 & 50 & 1 & 16 & + \\
\hline 14 & 3 & 1 & 33 & 1 & 33 & + \\
\hline Mean & & & 55 & & 39 & \\
\hline
\end{tabular}


No association was observed between the level of osteocalcin and sensitivity of the imaging modalities.

Also no correlation was noted between pain intensity and detectability in imaging modalities. In the case of patients with painless CRMO presenting solely with widening of the bone outline, radiological abnormalities were equally common in the analyzed imaging modalities.

\section{Discussion}

Chronic recurrent multifocal osteomyelitis is an atypical disease that is difficult to diagnose and thus often escapes detection or is misdiagnosed. One of the characteristic properties of the disease is the presence of several foci in the bone system; the foci may be clinically active or non-active. Thus, they may be "clinically silent". The WBMRI imaging modality suggested in recent publications makes it possible to search for such foci in the bone system. Therefore, performing the aforementioned examination extends the diagnostic management of the disease and may also allow for detection of other foci in the bone system, which might be an element of a clinical confirmation of CRMO diagnosis. Detecting the presence of more than one focus, and in particular of foci that are symmetrically situated in the bone system, suggests the diagnosis of CRMO in no uncertain terms [2, 10]. In turn, detection of radiologically active foci in the vertebral bodies is an indication for implementing CRMO therapy and allows avoidance of complications associated with clinical activation of foci $[9,11,12]$.

Another aim of the present WBMRI study was to assess the bone system with respect to the presence of other radiologically active and clinically inactive foci.

The results demonstrated that in patients with CRMO, the best correlation between the detected clinically active and radiologically active foci was achieved in scintigraphy.

The present authors determined a group of patients with multifocal CRMO (Table III) and, similarly as in the case of other investigators, they confirmed the observation of multifocal presence of abnormalities in the majority of patients - in the present material, this phenomenon was noted in $70 \%$ of the subjects $[13,14]$.

On the other hand, the present study did not confirm the extremely high detectability rate of disease foci or, in other words, sensitivity in detection of abnormalities in the bone system that was reported by other authors [14]. Possibly, the issue underlying the discrepancies is the method used in the study, e.g. model of MRI scanner. Perhaps other foci of bone abnormalities appear at various times in the course of the disease and this is a decisive factor in detecting their presence or absence.

Is seems, however, that particularly in view of recently published reports, whole-body MRI is diagnostically important; from the viewpoint of the present authors, it is worthwhile to repeat a similar study including larger groups of patients.

In conclusion, the results of the present study do not confirm the highest sensitivity of WBMRI in determining possible disease foci in CRMO as compared to other imaging modalities. Higher sensitivity in detection of radiologically active foci in CRMO has been noted in bone system scintigraphy. WBMRI allows for detecting the highest number of radiologically active foci when clinical activity is absent. During the 35-month follow-up, none of the WBMRI-detected radiologically active and clinically silent foci underwent clinical activation. The experience in employing whole body MRI presented in the literature and the present observations suggest that this examination should be performed in all patients diagnosed with CRMO in order to confirm the diagnosis.

\section{Conflict of interest}

The authors declare no conflict of interest.

\section{References}

1. Roderick MR, Shah R, Rogers V, Finn A, Ramanan AV. Chronic recurrent multifocal osteomyelitis (CRMO) advancing the diagnosis. Pediatr Rheumatol Online J 2016; 14: 47.

2. Fritz J, Tzaribachev N, Thomas C, et al. Magnetic resonance imaging-guided osseous biopsy in children with chronic recurrent multifocal osteomyelitis. Cardiovasc Intervent Radiol 2012; 35: 146-53.

3. Girschick HJ, Huppertz HI, Harmsen D, Krauspe R, Müller-Hermelink HK, Papadopoulos T. Chronic recurrent multifocal osteomyelitis in children: diagnostic value of histopathology and microbial testing. Hum Pathol 1999; 30: 59-65.

4. Fritz J, Tzaribatchev N, Claussen CD, Carrino JA, Horger MS. Chronic recurrent multifocal osteomyelitis: comparison of whole-body MR imaging with radiography and correlation with clinical and laboratory data. Radiology 2009; 252: 842-51.

5. Giedion A, Holthusen W, Masel LF, Vischer D. Subacute and chronic "symmetrical" osteomyelitis. Ann Radiol (Paris) 1972; 15: 329-42.

6. Strauss KJ, Kaste SC. The ALARA concept in pediatric interventional and fluoroscopic imaging: striving to keep radiation doses as low as possible during fluoroscopy of pediatric patients: a white paper executive summary. AJR Am J Roentgenol 2006; 187: 818-9.

7. Damasio MB, Magnaguagno F, Stagnaro G. Whole-body MRI: non-oncological applications in paediatrics. Radiol Med 2016; 121: 454-61.

8. Teixeira SR, Elias Junior J, Nogueira-Barbosa MH, Guimarães MD, Marchiori E, Santos MK. Whole-body magnetic resonance imaging in children: state of the art. Radiol Bras 2015; 48: 111-20. 
9. Arnoldi AP, Schlett CL, Douis H, et al. Whole-body MRI in patients with non-bacterial osteitis: radiological findings and correlation with clinical data. Eur Radiol 2017; 27: 2391-9.

10. Kennedy MT, Murphy T, Murphy M, Laffan E, Connolly P. Whole body MRI in the diagnosis of chronic recurrent multifocal osteomyelitis. Orthop Traumatol Surg Res 2012; 98: 461-4.

11. Armstrong A, Upadhyay N, Saxby E, Pryce D, Steele N. Chronic recurrent multifocal osteomyelitis causing an acute scoliosis. Case Rep Pediatr 2013; 2013: 649097.

12. Baulot E, Bouillien D, Giroux EA, Grammont PM. Chronic recurrent multifocal osteomyelitis causing spinal cord compression. Eur Spine J 1998; 7: 340-3.

13. Ferguson PJ, Sandu M. Current understanding of the pathogenesis and management of chronic recurrent multifocal osteomyelitis. Curr Rheumatol Rep 2012; 14: 130-41.

14. von Kalle T, Heim N, Hospach T, Langendörfer M, Winkler P, Stuber T. Typical patterns of bone involvement in whole-body MRI of patients with chronic recurrent multifocal osteomyelitis (CRMO). Rofo 2013; 185: 655-61. 